\title{
LBPV descriptors-based automatic ACR/BIRADS classification approach
}

\author{
Alima Damak Masmoudi ${ }^{1 *}$, Norhen Gargouri Ben Ayed ${ }^{1}$, Dorra Sellami Masmoudi ${ }^{1}$ and Riad Abid ${ }^{2}$
}

\begin{abstract}
Mammogram tissue density has been found to be a strong indicator for breast cancer risk. Efforts in computer vision of breast parenchymal pattern have been made in order to improve the diagnostic accuracy by radiologists. Motivated by recent results in mammogram tissue density classification, a novel methodology for automatic American College of Radiology Breast Imaging Reporting and Data System classification using local binary pattern variance descriptor is presented in this article. The proposed approach characterizes the local density in different types of breast tissue patterns information into the LBP histogram. The performance of macro-calcification detection methods is developed using FARABI database. Performance results are given in terms of receiver operating characteristic. The area under curve of the corresponding approach has been found to be $79 \%$.
\end{abstract}

Keywords: Mammogram, Breast tissue, Texture, Classification, Feature extraction, Macro-calcification detection, LBP, LBPV, ROC, ACR/BIRADS, CAD, ANN

\section{Introduction}

Female breast cancer is a common cause of cancerrelated deaths in women, especially in western countries and where statistics are available. Mammographic images are hard to interpret because of the textural morphology information complexity of the breast and the number of image parameters that affect the acquisition of mammograms [1].

In the evaluation of mammogram images, CAD (Mammographic Computer-Aided Diagnosis) systems are aimed at assisting radiologists [2,3]. Studies in CAD systems tend to concentrate on the detection and classification of mammographic masses and micro-calcification [4]. In addition, recent research has shown that the sensitivity of these systems to detect masses in mammograms is significantly decreased as the density of the tumors increases [5]. These classification is based on both severity for the disease and image properties.

Classification with textural mammogram information can be based on a number of categories that might not explain the same mammographic features [4-8]. ACR/BIRADS classification [7] is becoming a standard

*Correspondence: damak_alima@yahoo.fr

${ }^{1}$ Computers Imaging and Electronics Systems (CIELS), University of Sfax,

Sfax Engineering School, Sfax-Tunisia, BP W, 3038 Sfax, Tunisia

Full list of author information is available at the end of the article in the assessment of mammogram images, which are classified in fourth categories according to their density (Figure 1).

- ACR/BIRADS I: the breast is almost entirely fatty.

- ACR/BIRADS II: there is some fibro-glandular tissue.

- ACR/BIRADS III: the breast is heterogeneously dense.

- ACR/BIRADS IV: the breast is extremely dense.

It is well known that there is a strong correlation between textural density in mammographic image and the risk of developing tumors [8]. Figure 1 shows four samples in the American College of Radiology's Breast Imaging Reporting and Data System (ACR/BIRADS) classes with respect to density.

ACR/BIRADS classification will be beneficial, to characterize what the mammogram density is since it is a parameter criterion in Breast Imaging Reporting and Data System classification, as well as to create an optimal approach to follow if, for example, detecting masses in mammographic image of tissue abnormality detection (marcocalcification/microcalcification). An early step of density classification will switch on for each class, this step is more efficient for segmentation approach. For example, detecting a macrocalcification in surrounding darker pixels as in the first class could be done differently if neighbors are brighter as in the third or four class.

\section{Springer}



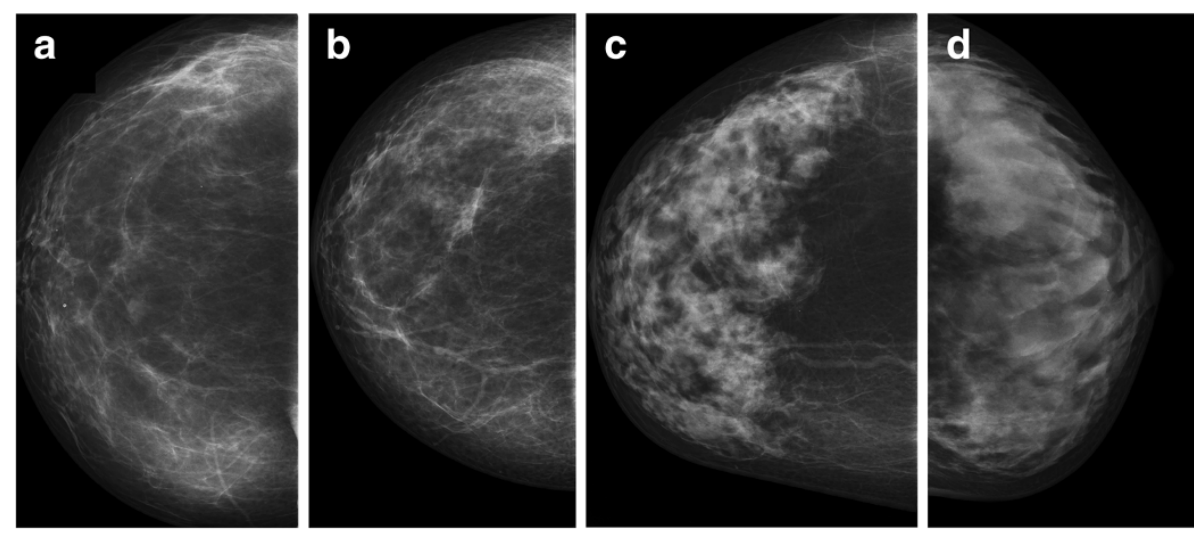

Figure 1 Four samples in the ACR/BIRADS classes with respect to density: (a) ACR/BIRADS I, (b) ACR/BIRADS II, (c) ACR/BIRADS III, (d) ACR/BIRADS IV.

In this article, a novel approach to automatic breast tissue classification is investigated. The first step of the proposed approach is a pre-processing denoising module. In the next step, texture features are extracted using a novel descriptor named local binary pattern variance (LBPV). Performance evaluation is based on testing the algorithm on images from a new Tunisian database located at the radiology center EL FARABI Sfax Tunisia. Then, an artificial neural network (ANN) is used for classifying the breast density tissue. To highlight the interest of such classification, a brief summary on methods in segmentation masses region for each density class is provided.

In Section 2, we briefly describe our image processing and classification system. Then, we present the proposed textural feature extraction using LBPV descriptor and we provide a brief summary of the breast segmentation approaches. In Section 3, we present the performances results followed by conclusion in Section 4.

\section{System module description}

The functionality of the proposed system is provided by means of four modules: (a) the preprocessing module, (b) the feature extraction module, (c) the ANN classification module, and (d) the mass segmentation (Figure 2).

\subsection{Preprocessing}

In this module, input images are prepared for the processing steps that will follow. The basic need for preprocessing in mammographic images is to increase the contrast, especially for dense breasts.

To remove the background noise [9], preserving the local information of suspicious areas can enhance mammograms. This approach was proposed by Lai et al. [10], who used modified median filtering and four selective averaging schemes called selective median filter. The output of this filter within a window $W(n, m)$, centered at image coordinates $(n, m)[11]$ is given by

$$
I_{n, m}=\operatorname{median}\left\{\begin{array}{c}
I_{r, s}:(r, s) \in N(n, m) \\
\left|I_{r, s}-I_{n, m}\right|<I_{\mathrm{th}}
\end{array}\right.
$$

where $I_{n, m}$ is the image intensity at $(n, m), N(n, m)$ is the area in the image covered by the window $W(i, j)$, and $I_{\text {th }}$ is the threshold.

The set of pixels, in computing the median, is restricted to those with a difference in gray level not greater than a some threshold $I_{\text {th }}$. Adjusting the parameter $I_{\text {th }}$ allows to control the amount of edge smearing and to remove the background noise [12].

\subsection{A novel textural feature extraction using LBPV descriptor}

LBP [13] is a gray scale operator which characterizes the textural structure in the image. A pattern number is computed by comparing a central pixel in the gray image value with its neighborhoods:

$$
\begin{aligned}
& \operatorname{LBP}_{P, R}=\sum_{P=0}^{P-1} \operatorname{Sign}\left(g_{p}-g_{c}\right) 2^{P} \\
& \operatorname{Sign}(x)=\left\{\begin{array}{l}
1, x \geq 0 \\
0, x<0
\end{array}\right.
\end{aligned}
$$

where $g_{c}$ is the gray value of the central pixel, $g_{p}$ is the value of its neighbors, $P$ is the number of neighbors, and $R$ is the radius of the neighborhood.

If the coordinates of $g_{c}$ are $(0,0)$, then the coordinates of $g_{p}$ are given by $\left(-R \sin \left(\frac{2 \pi p}{P}\right), R \cos \left(\frac{2 \pi p}{P}\right)\right)$. The gray values of neighbors which do not fall exactly in the center of pixels are estimated by interpolation. 


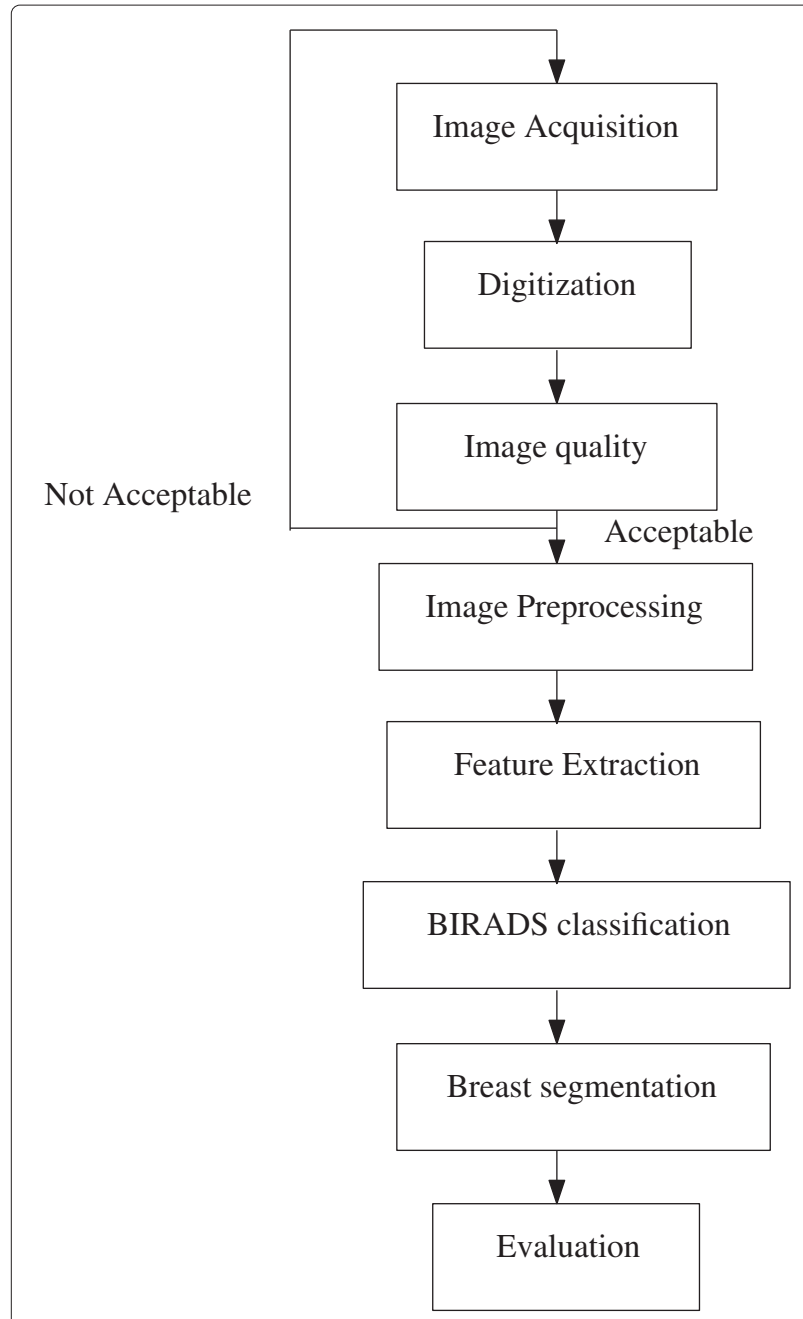

Figure 2 The proposed method of macro-calcification detection.

Suppose the texture image is $N * M$. After identifying the LBP pattern of each pixel $(i, j)$, the whole texture image is represented by building a histogram:

$$
\begin{aligned}
& H(k)=\sum_{i=1}^{N} \sum_{j=1}^{M} f\left(L B P_{P, R}(i, j), k\right), \quad k \in[0, K] \\
& f(x, y)=\left\{\begin{array}{l}
1, x=y \\
0, \text { otherwise }
\end{array}\right.
\end{aligned}
$$

To remove the effect of rotation in local image the $\mathrm{LBP}_{P, R}^{r i}$ (rotation invariant local binary pattern) is defined by:

$$
\operatorname{LBP}_{P, R}^{r i}=\min \left\{\operatorname{ROR}\left(\operatorname{LBP}_{P, R}, i\right) \mid i=0,1, \ldots, P-1\right\}
$$

where $\operatorname{ROR}(x, i)$ is a circular bit-wise right shift on the $P$-bit number.
The rotation invariance with uniform patterns $\mathrm{LBP}_{P, R}^{r i u 2}$ is proposed by Ojala et al. [13].

$$
\operatorname{LBP}_{P, R}^{r i u 2}= \begin{cases}\sum_{p=0}^{p-1} \operatorname{sign}\left(g_{p}-g_{c}\right), \text { if } U\left(\operatorname{LBP}_{P, R}\right) \leq 2 \\ p+1, \quad \text { otherwise }\end{cases}
$$

where superscript riu 2 reflects the use of rotation invariant uniform patterns, $U$ introduces the uniformity measure

$$
U\left(\mathrm{LBP}_{P, R}\right)=\left|\begin{array}{l}
\operatorname{sign}\left(g_{p-1}-g_{c}\right)-\operatorname{sign}\left(g_{0}-g_{c}\right) \\
+\sum_{p=1}^{p-1} \operatorname{sign}\left(g_{p}-g_{c}\right)-\operatorname{sign}\left(g_{p-1}-g_{c}\right)
\end{array}\right| .
$$

A rotation invariant variance measures (VAR)of the local image can be defined as follows:

$$
\operatorname{VAR}_{P, R}=\frac{1}{p} \sum_{p=0}^{p-1} \operatorname{sign}\left(g_{p}-\mu\right)^{2}
$$

where

$$
\mu=\frac{1}{p} \sum_{p=0}^{p-1} \operatorname{sign}\left(g_{p}\right)
$$

$\frac{\mathrm{LB}_{P, R}^{r i u 2}}{\operatorname{VAR}_{P, R}}$ is the powerful descriptor of local contrast information because it exploits the complementary characteristics of local spatial patterns and local contrast [13].

Usually high-frequency textural information regions will have higher variances and contribute more to the discrimination of images. Therefore, LBPV is proposed to characterize the local contrast information into a onedimensional LBP histogram where $\operatorname{VAR}_{P, R}$ can be used as an adaptive weight to adjust the contribution of LBP descriptor in histogram calculation. The local binary variance code histogram is computed as follows:

$$
\begin{aligned}
& \operatorname{LBPV}_{P, R}=\sum_{i=1}^{N} \sum_{j=1}^{M} w\left(\operatorname{LBP}_{P, R}^{r i u 2}(i, j), k\right), \quad k \in[0, K] \\
& w\left(\operatorname{LBP}_{P, R}^{r i u 2}(i, j), k\right)=\left\{\begin{array}{l}
\operatorname{VAR}_{P, R}(i, j), \operatorname{LBP}_{P, R}^{r i u 2}(i, j)=k \\
0 \quad \text { otherwise }
\end{array}\right.
\end{aligned}
$$

Though locally operated, LBPV operator has an excellent ability to summarize the different global or local densities in the image. Figure 3 illustrates that each density in the image can be illustrated by a respective peak in the occurrence histogram. Therefore, LBPV can be seen as a good descriptor candidate that could eventually 

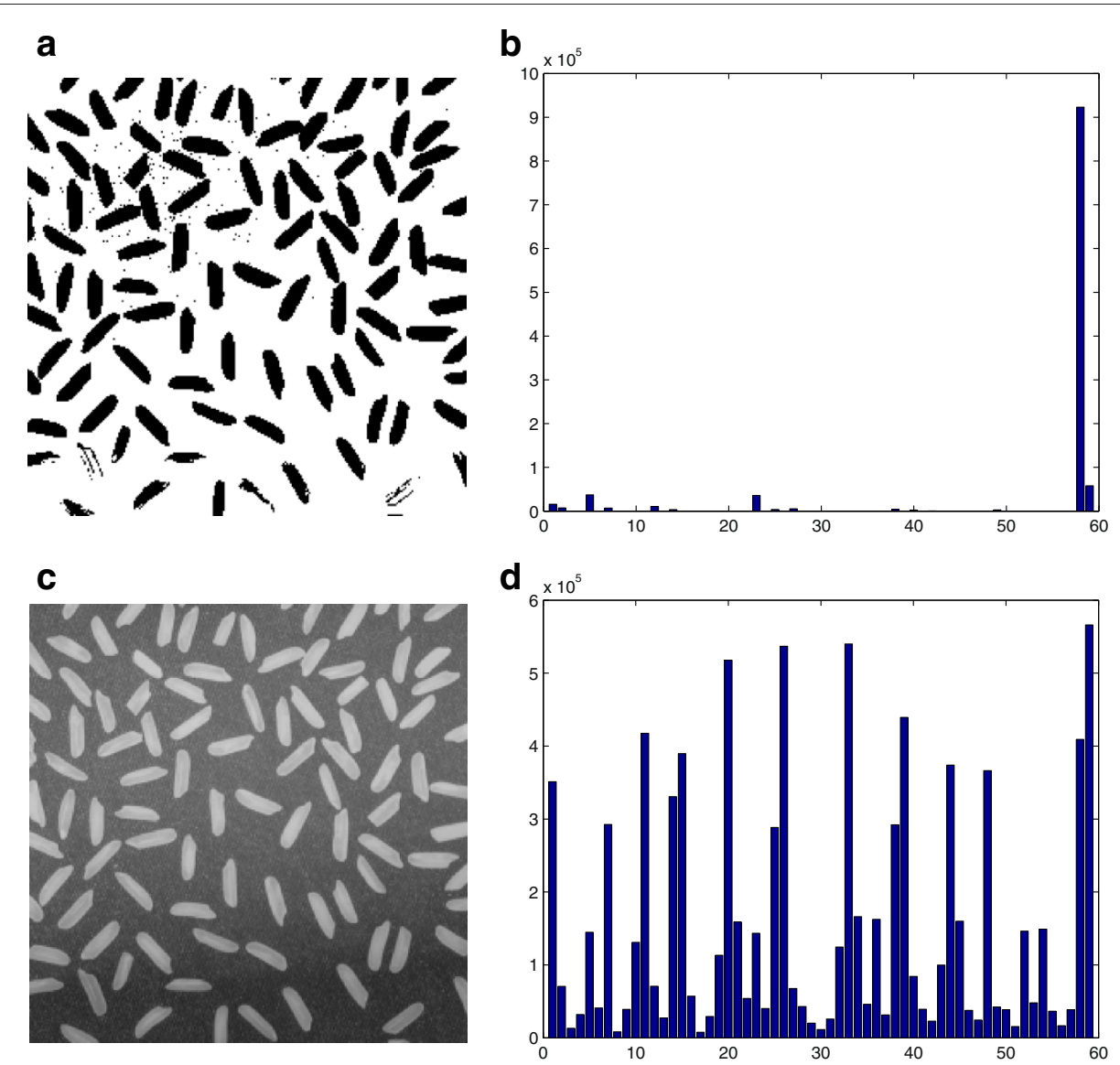

Figure 3 Perfect ability of LBPV operator to describe global density in the image: (a) Binary image, (b) Histogram LBPV of binary image, (c) Gray level image, (d) Histogram LBPV of gray level image.

mirror and handle sensitively different densities in mammographic images.

The LBPV-based descriptor operator is a good contrast distribution method. Two occurrence histograms are, respectively, generated. Taking $R=1$ and $P=8$, the size of the vector is fixed to 512. This size is unique and independent of minimum number or image size. Such advantage overcomes limitations of direct matching discrepancies. Figure 4 illustrates a mammogram sample image with the corresponding histogram.

\subsection{Breast tissue density classification module with ANN} Usually, ANNs can be considered as an information of system which is composed of interconnected elements constituting a network. Inspired by the biological nervous system, ANN adjusts weights between neurons (Table 1).

Let $x=\left(x_{1}, x_{2}, \ldots, x_{d}\right)^{T}$ be an input vector and $w=$ $\left(w_{1}, w_{2}, \ldots, w_{d}\right)^{T}$ the weight vector, the output is determined as follows:

$$
y=g\left(w^{T} x_{b}\right)=g\left(\sum_{i=1}^{d} w_{i} x_{i}-b\right)
$$

where $g($.$) is namely a sigmoidal activation function$ defined by

$$
g(x)=\left(1+e^{-x}\right)^{-1}
$$

For each mammogram samples, LBPV vectors are computed. The description based on these features is used in the recognition step as neural network inputs. The neural network architecture is represented in Figure 5.

To be able to evaluate the effectiveness of the training, one can measure the relative error as follows:

$$
E=\frac{\sum_{i=1}^{n}\left(I_{N}-I_{T}\right)}{\text { Number of samples }},
$$

where $I_{N}$ is the image resulting from ANN output and $I_{T}$ is the target.

After training steps, generalization error was evaluated for different features and network conditions. Figure 6 shows the evolution of training and generalization errors by incrementing the number of hidden layers. 

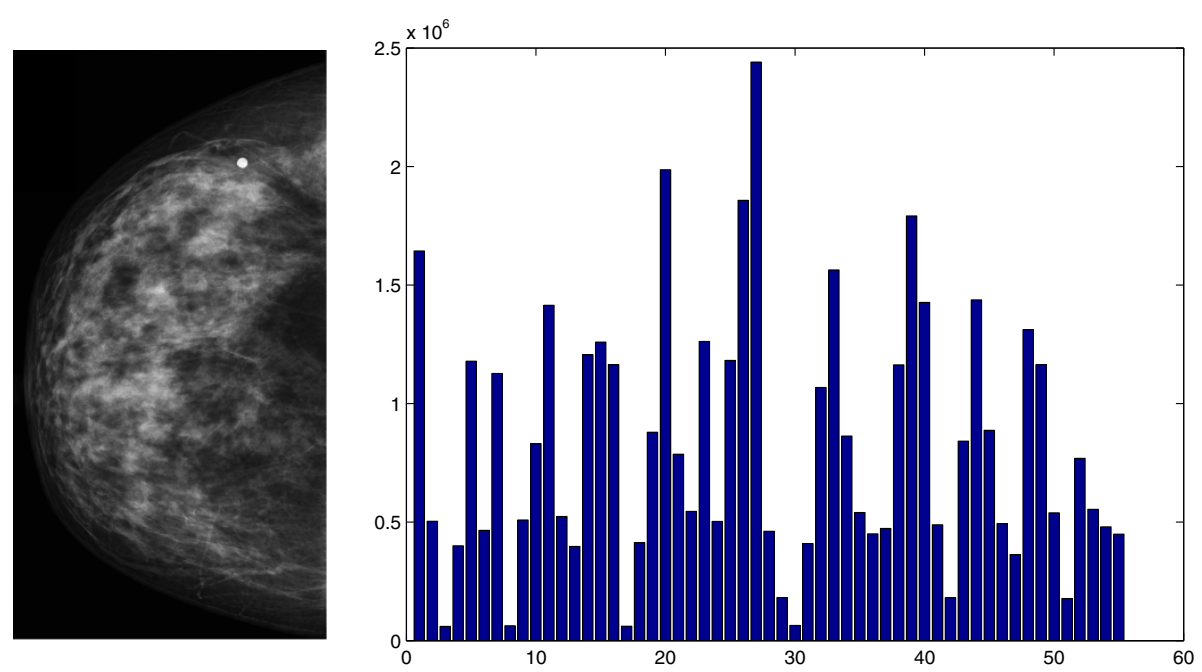

Figure 4 Perfect ability of LBPV operator to describe global densities in the image.

\subsection{Breast segmentation}

In general, masses in low-density breasts are better detected than masses in high-density breasts, although each algorithm performs differently with regard to this.

\subsubsection{Detection of concentric layers}

In [14], the segmentation of masses by detection of concentric layers, using progressively lower average intensity, is proposed. This approach with a region granulation (i.e., a grey-level transformation) reduces the large number of intensity levels.

The grey-level transformation step starts by linearly normalizing the intensity between levels 0 and 1. Next, the pixels are assigned a grey level. This is done by sequentially visiting each granule pixel and examining its local neighborhood. If all neighbors are within $98 \%$ of the granularity, they are assigned to the same grey level, else they will be assigned to a different granulation. After this transformation, a morphological opening is performed to decrease scattered grey levels.

The segmentation of suspicious masses regions is based on the inspection of the granularity. Thus, all the regions with similar or higher levels are grouped. For each level, a set of features, including area, eccentricity, solidity, and dispersion, are computed. This procedure is repeated for the brightest levels. Therefore, the region

\section{Table 1 Output of ANN classifier}

\begin{tabular}{llll}
\hline Output & $\boldsymbol{y 3}$ & $\boldsymbol{y} \mathbf{2}$ & $\boldsymbol{y} \mathbf{1}$ \\
\hline ACR/BIRADS I & 0 & 0 & 1 \\
ACR/BIRADS II & 0 & 1 & 0 \\
ACR/BIRADS III & 0 & 1 & 1 \\
ACR/BIRADS VI & 1 & 0 & 0 \\
\hline
\end{tabular}

growing is established, and the suspicious regions are those containing at least three developing concentric layers. All the parameters used have empirically been adjusted to FARABI database. One of these parameters is related to the minimum distance between possible macrocalcifications. This distance will be used later to obtain probability images.

\subsubsection{Thresholding approach}

This approach is proposed by Kom et al. [15]. The corresponding algorithm is based on the thresholding mammographic image obtained by subtracting from the mammogram a linear filtered representation of itself.

The enhanced image $I_{\mathrm{EN}}$ is given for each coordinates $(x, y)$ by:

$I_{\mathrm{EN}}(x, y)=a \times \log \left(1+m \times I_{m}(x, y)\right), I_{m}(x, y)<\alpha$

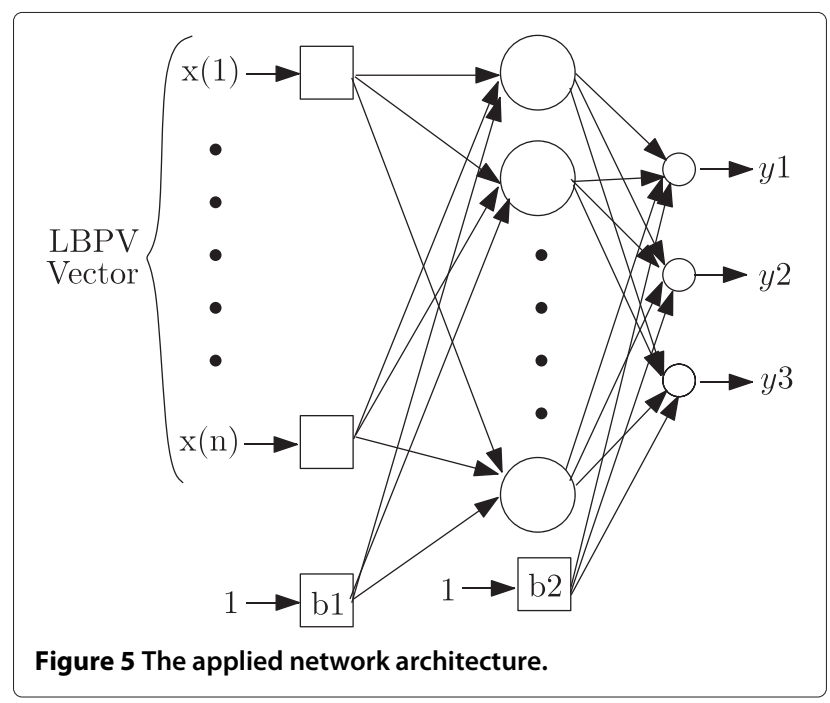




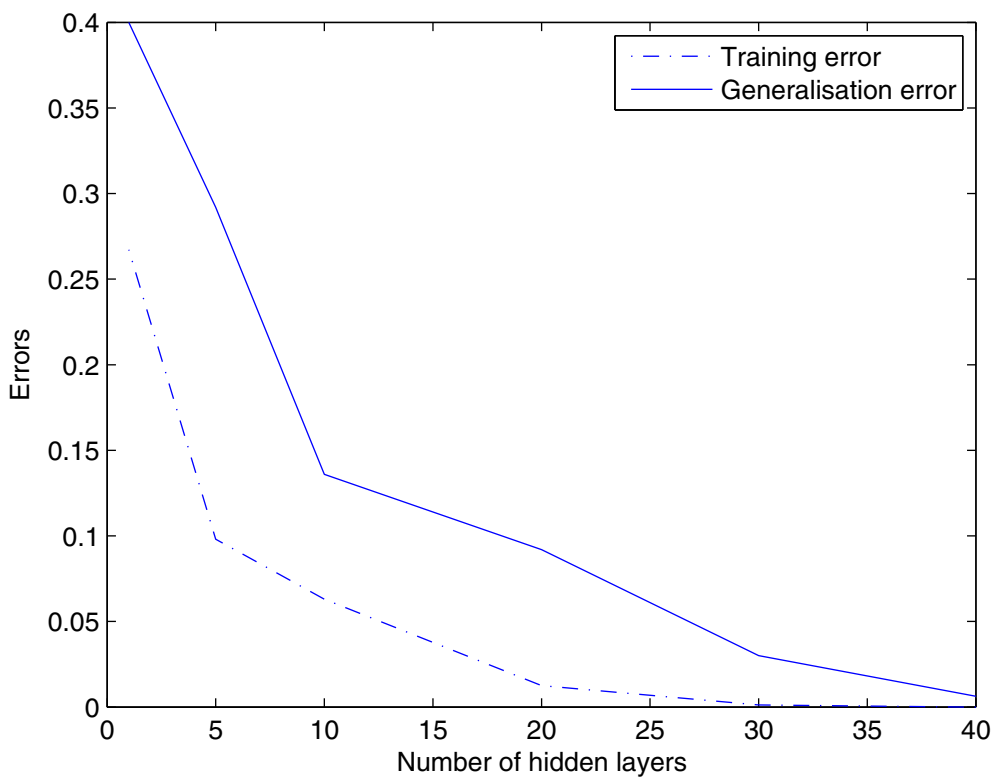

Figure 6 Evolution of training and generalization errors by increasing the number of hidden layers.

$$
I_{\mathrm{EN}}(x, y)=\frac{\exp \left(\frac{I_{m}(x, y)}{a}\right)-1}{b}, I_{m}(x, y)>\alpha
$$

where $I_{m}(x, y)$ is the original mammogram, $m$ is its maximum grey level, $b=\frac{1-\exp \left(\frac{m}{a}\right)}{m}, a$ and $\alpha$ are two parameters fixed experimentally; in this study, $a=10$ and $\alpha=0.3$.

Consequently, the subtracted image $I_{\text {Sub }}$ is given for each pixel $(x, y)$ by:

$$
I_{\mathrm{Sub}}(x, y)=I_{m}(x, y)-I_{\mathrm{EN}}(x, y)
$$

At last, the subtracted image is thresholded by using an adaptive local threshold to obtain suspicious macrocalcifications.

\subsubsection{Laplacian edge detector approach}

This method is proposed by Petrick et al. [16] who used an optimal Laplacian Gaussian edge detector (LGED) with the aim of finding closed regions in the enhanced version of the mammographic image. This approach begins by preprocessing the mammogram using a density-weighted contrast enhancement (DWCE) filter, which is based on two filtered mammograms of the original image $I_{m}(x, y)$ : the first is the density image $I_{F_{D}}(x, y)$, which is a smoothed version of the image, obtained by using a Gaussian filter. The next is the contrast image $I_{F_{C}}(x, y)$, obtained by subtracting the original mammogram from a second smoothed version of the image.

The density of mammograms is filtered all over again with a nonlinear filter $K_{M}$ and used to define a multiplication factor which adjusts the related pixel in the contrast image. Thus, the density parameter of each pixel is weighted by local contrast. The intermediate image $I_{F_{K C}}$ can be expressed by

$$
I_{F_{K C}}(x, y)=K_{M}\left(I_{F_{D}}(x, y)\right) * I_{F_{C}}(x, y)
$$

where $I_{F_{K C}}(x, y)$ is used to define a next multiplication value using another nonlinear filter $K_{N L}$, which is multiplied by the weighted contrast of the related pixels

$$
I_{F_{E}}(x, y)=K_{N L}\left(I_{F_{K C}}(x, y)\right) * I_{F_{K C}}(x, y)
$$

where $I_{F_{E}}(x, y)$ is the output of the DWCE filter. The output of this filtering process is a mammogram where the potential masses are highlighted.

To detect macro-calcifications, an edge detector is used. An LGED was applied. It is defined by

$$
\operatorname{LGED}(x, y)=\nabla^{2} G(x, y) * I_{F_{E}}(x, y),
$$

where $G(x, y)$ is a two-dimensional Gaussian smoothing function.

\subsubsection{Classifier approach}

This method is proposed by Karssemeijer and te Brake $[17,18]$. The classification approach makes possible the detection of macro-calcifications using second-order Gaussian derivative operators. If a line-like structure is present at a given site, this algorithm provides an estimation of the orientation of textural mammogram tissue. With this information, two new features are built. The first one characterizes the total number of pixels pointing the center, while the next feature estimates whether 
these directions are circularly oriented. With both features, and a set of classified mammograms, this approach trains a binary decision tree. Afterward, the decision tree can be used for macro-calcification detection in medical image.

\section{System performances}

In order to test the proposed method, images from EL FARABI database are used. Images in this database have their density classified according to ACR/BIRADS categories.

\subsection{EL FARABI database}

The mammograms used in this study were collected from EL FARABI radiologic center. They are acquired using different mammographic screen/film system and settings (all with molybdenum anode) and in the framework of different applications. The obtained images including both clinical routine carried out on symptomatic women and screening programs addressed to asymptomatic women. All the images were digitized with a CCD scanner at a pixel size of $85 \times 85 \mu \mathrm{m}^{2}$ with a 12-bit resolution. Each image is thus $3560 \times 4640$ pixels with $G=2^{12}=4096$ gray-level tones. No normalization was applied to images. The database consists of 2052 mammograms from 342 analyzed subjects. Some of the mammograms show different views (craniocaudal, lateral, oblique) of the same subject and are considered as different samples in our analysis. The diagrams reported in Figure 7 show the partition of the database in left/right breast images (left) and craniocaudal/oblique/ lateral views (right).

\subsection{Receiver operating characteristics (ROC)}

ROCs are usually used in many fields for decision making to validate a given classification method. In this study, we use it for a validation of ACR/BIRADS mammogram density classification.

\subsubsection{ROC curves}

An ROC curve is a graphical visualization of the TPR (True Positive Rate) as a function of the FPR (False Positive Rate) of mammogram classifier systems.

\subsubsection{The ROC convex hull method}

The ROCCH (ROC convex hull) method accommodates both binary and continuous ROC curve. Binary recognitions are represented by individual points in ROC space. Continuous ROC produces numeric outputs of thresholds that can be applied, yielding a series of (FPR,TPR) pairs forming an ROC curve. Each point may or may not contribute to the ROC convex hull.

\subsubsection{Area under ROC curve}

The area under the ROC curve (AUC) is common metric that can be used for performance evaluation of a decisionmaking system. It constitutes an efficient way to compare different tests (indicator variables). For performance evaluation, it is convenient to reduce the ROC curve to a single scalar value representing expected performance. The easiest possibility is to calculate the AUC which is a part of the area of the unit square. Consequently, the value of AUC will always satisfy the following inequalities

$$
0 \leq \mathrm{AUC} \leq 1
$$

It is clear that if the AUC is close to 1 (area of unit square), AUC indicates very good diagnostic test. However, as the random guessing produces the diagonal line between the points $[0,0]$ and $[1,1]$, which has an area of 0.5 , reasonable tests should have

$$
0.5 \leq \mathrm{AUC} \leq 1
$$

The AUC has an important statistical property: the AUC of an $\mathrm{ROC}$ relative to a recognition system is equivalent to
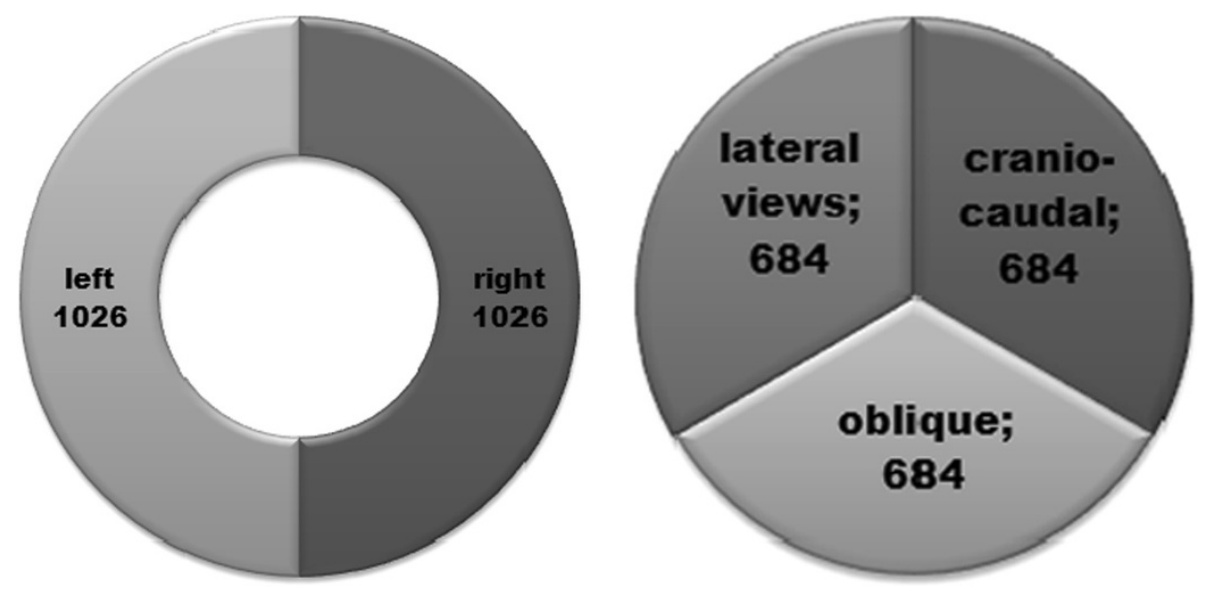

Figure 7 Database partition. Left: number of left/right breast images. Right: number of craniocaudal/oblique/lateral views. 


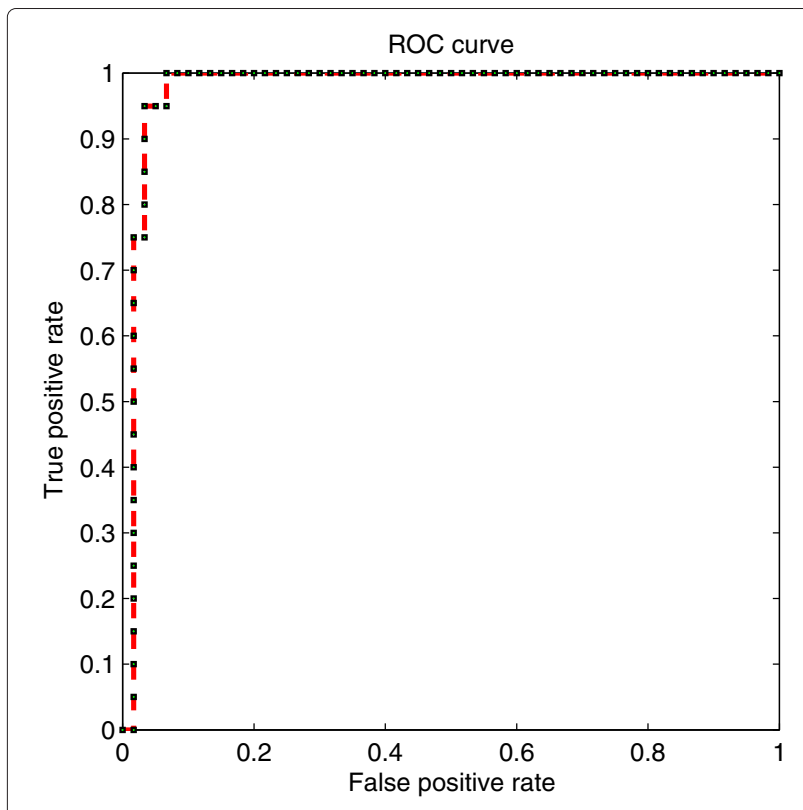

Figure 8 ROC curve for ACR/BIRADS automatic classification.

the probability that the recognition will evaluate randomly chosen positive instance higher than a randomly chosen negative instance.

\subsection{ACR/BIRADS automatic classification results}

The method was applied to a set of 400 image mammographic taken from the FARABI Digital Database. This database provides for each mammogram additional information, including the density of the breast determined by an expert according to BIRADS categories. In order to simulate the real world, our database is formed by 50 mammograms with ACR/BIRADS I, II, III, and IV, so 200 images are used for training. However, 200 mammographic image are used for generation test.

Figure 8 shows that the AUC of this approach has a value of 0.97 .

\subsection{Breast tissue influence}

The breast density evaluation experiment is related to the ability of each method to detect macro-calcification in all FARABI database images. This evaluation mimics the radiologist in identifying the presence of tumors.

The accuracy of the approach is depending on breast tissue density classification and is summarized in Table 2. This table shows the AUC for each method detailed for each ACR/BIRADS density. Generally, macrocalcification are detected in low-density images than in high-density images.

For instance, looking at experimental results, algorithms for detection of concentric layers have better performance on fatty breasts tissues on ACR/BIRADS I compared to other density classes. Therefore, thresholding and Laplacian edge detector approaches get the best accuracy for mammographic images belonging to ACR/BIRADS II and ACR/BIRADS III, respectively. Classifier approach performs better for the most dense tissue (ACR/BIRADS IV).

The reason for such different behaviors is related to different factors. For example, macro-calcifications in fatty mammograms frequently have a more delineated boundary than in denser medical images. Moreover, one can see a set of circumscribed layers around the macro-calcifications that are exploited in the granularity algorithm (i.e., detection of concentric layers). Thresholding and Laplacian edge detector approaches seem beneficial for medical images belonging to intermediate ACR/BIRADS II and ACR/BIRADS III classes, where macro-calcifications are highlighted with respect to the normal tissue. To end with classifier approach which performs better for the dense tissues (ACR/BIRADS IV). In fact, it uses the contour information for masse detection and it has better performances for increased intensity changes in mammograms.

\section{Conclusion}

A novel automatic ACR/BIRADS classification for segmentation of mammographic masses is presented. To exploit the local and global textural information in mammographic images, the LBPV was proposed. This descriptor characterizes globally rotation invariant matching with locally variant LBP features for mammogram texture classification. This approach is tested on 342 pairs of patient mammograms.

Table 2 Influence of the breast density based on AUC

\begin{tabular}{lllll}
\hline & $\begin{array}{l}\text { Detection of } \\
\text { concentric layers }\end{array}$ & $\begin{array}{l}\text { Thresholding } \\
\text { approach }\end{array}$ & $\begin{array}{l}\text { Laplacian edge } \\
\text { detector approach }\end{array}$ & $\begin{array}{c}\text { Classifier } \\
\text { approach }\end{array}$ \\
\hline ACR/BIRADS I & 0.81 & 0.71 & 0.70 & 0.72 \\
ACR/BIRADS II & 0.64 & 0.79 & 0.69 & 0.65 \\
ACR/BIRADS III & 0.59 & 0.61 & 0.74 & 0.63 \\
ACR/BIRADS VI & 0.52 & 0.58 & 0.59 & 0.78 \\
\hline
\end{tabular}


As performances metric, we get for the EL FARABI database an AUC which has value of 0.79 .

A segmentation technique has also been done, describing several methods and pointing out their specific features. These approaches have fully been evaluated using ROC curve analysis and tested using a digitized database. Annotations used as the gold standard were provided by expert radiologists who read mammograms routinely.

Segmentation results depend on the breast density. Based on our testing of these algorithms on the FARABI database, abnormal mammograms belonging to ACR/BIRADS I tend to show improved detection over abnormal mammograms belonging to other ACR/BIRADS category. This is related to the increase of the contrast in parenchymal tissue, which is mistaken for abnormal regions.

\section{Competing interests}

The authors declare that they have no competing interests.

\section{Author details}

${ }^{1}$ Computers Imaging and Electronics Systems (CIELS), University of Sfax, Sfax Engineering School, Sfax-Tunisia, BP W, 3038 Sfax, Tunisia. ${ }^{2}$ EL FARABI Radiology Center, Rue Ahmed Essikilli Imm. El Farabi Sfax El Jadida,

Sfax-Tunisia, BP W, 3003 Sfax, Tunisia.

Received: 28 November 2012 Accepted: 11 March 2013

Published: 17 April 2013

\section{References}

1. JW Byng, NF Boyd, E Fishell, RA Jong, MJ Yaffe, Automated analysis of mammographic densities. Phys. Med. Biol. 41, 909-923 (1996)

2. RL Birdwell, DM Ikeda, KD O'Shaughnessy, EA Sickles, Mammographic characteristics of 115 missed cancers later detected with screening mammography and the potential utility of computer-aided detection. Radiology. 219, 192-202 (2001)

3. TW Freer, MJ Ulissey, Screening mammography with computeraided detection: prospective study of 01286 patients in a community breast center. Radiology. 220, 781-786 (2001)

4. L Tabar, T Tot, PB Dean, Breast Cancer-The Art and Science of Early Detection With Mammography. (Georg Thieme Verlag, Stuttgart, Germany, 2005)

5. WT Ho, PWT Lam, Clinical performance of computer-assisted detection (CAD) system in detecting carcinoma in breasts of different densities. Clin. Radiol. 58, 133-136 (2003)

6. NF Boyd, JW Byng, RA Jong, EK Fishell, LE Little, AB Miller, GA Lockwood, DL Tritchler, MJ Yaffe, Quantitative classification of mammographic densities and breast cancer risk: results from the Canadian national breast screening study. J. Nat. Cancer Inst. 87, 670-675 (1995)

7. American College of Radiology, Illustrated Breast Imaging Reporting and Data System BIRADS, 3rd edn. (American College of Radiology, Philadelphia, PA, 1998)

8. JN Wolfe, Risk for breast cancer development determined by mammographic parenchymal pattern. Cancer. 37, 2486-2492 (1976)

9. S-C Yang, C-M Wang, Y-N Chung, G-C Hsu, S-K Lee, P-C Chung, C-I Chang, A computer aided system for mass detection and classification in digitized mammograms. Biomed. Eng.- Appl. Basis Commun. 17, 215-228 (2005)

10. S Lai, X Li, W Bischof, On techniques for detecting circumscribed masses in mammograms. IEEE Trans. Med. Imagi. 8, 377-386 (1989)

11. RC Gonzalez, Digital Image Processing Using Matlab. (Pearson Publication, 2005)

12. MP Sampat, MK Markey, AC Bovik, Computer-Aided Detection and Diagnosis in mmammography. (Elsevier Academic Press, Amsterdam, 2005), pp. 1195-1217
13. T Ojala, M Pietikäinen, TT Mäenpää, Multiresolution gray-scale and rotation invariant texture classification with local binary pattern. IEEE Trans. Pattern Anal. Mach. Intell. 24(7), 971-987 (2002)

14. NH Eltonsy, GD Tourassi, AS Elmaghraby, A concentric morphology model for the detection of masses in mammography. IEEE Trans. Med. Imagi. 26(6), 880-889 (2007)

15. G Kom, A Tiedeu, M Kom, Automated detection of masses in mammograms by local adaptive thresholding. Comput. Biol. Med. 37(1), 37-48 (2007)

16. N Petrick, HP Chan, B Sahiner, D Wei, An adaptive density-weighted contrast enhancement filter for mammographic breast mass detection. IEEE Trans. Med. Imagi. 15(1), 59-67 (1996)

17. N Karssemeijer, GM te Brake, Detection of stellate distortions in mammograms. IEEE Trans. Med. Imagi. 15(5), 611-619 (1996)

18. N Karssemeijer, GM te Brake, Combining single view features and asymmetry for detection of mass lesions. Digital Mammography. 25, 95-102 (1998)

doi:10.1186/1687-5281-2013-19

Cite this article as: Masmoudi et al:: LBPV descriptors-based automatic ACR/BIRADS classification approach. EURASIP Journal on Image and Video Processing 2013 2013:19.

\section{Submit your manuscript to a SpringerOpen ${ }^{\mathcal{O}}$ journal and benefit from:}

- Convenient online submission

- Rigorous peer review

- Immediate publication on acceptance

- Open access: articles freely available online

- High visibility within the field

- Retaining the copyright to your article

Submit your next manuscript at $>$ springeropen.com 